\title{
La représentation de la biodiversité africaine au Musée Royal de l'Afrique Centrale : la muséologie du compromis
}

Sabine van der Hoorn

\section{(2) OpenEdition}

\section{Journals}

Édition électronique

URL : https://journals.openedition.org/ere/4390

DOI : $10.4000 /$ ere.4390

ISSN : 2561-2271

Éditeur

Centr'ERE

Référence électronique

Sabine van der Hoorn, « La représentation de la biodiversité africaine au Musée Royal de l'Afrique

Centrale : la muséologie du compromis », Éducation relative à l'environnement [En ligne], Volume 15 - 1 |

2019, mis en ligne le 20 décembre 2019, consulté le 15 octobre 2021. URL : http://

journals.openedition.org/ere/4390; DOI : https://doi.org/10.4000/ere.4390

Ce document a été généré automatiquement le 15 octobre 2021

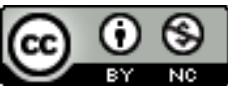

La revue Éducation relative à l'environnement est mise à disposition selon les termes de la Licence Creative Commons Attribution - Pas d'Utilisation Commerciale 4.0 International. 


\title{
La représentation de la biodiversité africaine au Musée Royal de l'Afrique Centrale : la muséologie du compromis
}

\author{
Sabine van der Hoorn
}

1 Fin 2018, le Musée Royal de l'Afrique Centrale de Belgique (MRAC), nouvellement baptisé Africamuseum ${ }^{1}$, a rouvert ses portes. Fermé pendant plus de 5 ans pour des travaux de rénovation sans précédent, le musée présente aujourd'hui la culture et la nature africaines sous un nouveau jour. En effet, une nouvelle étape vient d'être franchie quant à la représentation de la nature africaine depuis son ouverture initiale en 1910.

2 Si le bâtiment et les collections datant de l'époque coloniale ont toujours été réutilisés durant ces cent années de fonction, la vision de la nature et le regard portés sur les collections zoologiques ont sans cesse évolué. Considérées initialement comme des trophées ou témoins des chasses coloniales, les collections zoologiques sont de nos jours davantage perçues comme des réserves d'objets scientifiques et tendent à représenter une ressource alimentaire et marchande très convoitée et menacée.

3 Aujourd'hui, les visiteurs découvrent un «patchwork» muséologique que nous qualifions de muséologie du compromis. En effet, autour des anciennes pièces de collection, notamment les animaux naturalisés, s'est construit un discours polyphonique: celui de l'équipe de conception, mais aussi d'autres professionnels comme les scénographes et les conseillers issus de la diaspora. Différentes conceptions de musées en ont résulté, et leur juxtaposition a parfois conduit à un manque de lisibilité du discours.

4 Dans cet article, nous allons tout d'abord analyser, de 1910 à 2018, les divers modes d'utilisation des pièces d'expositions qui avaient pour finalité de présenter les différentes composantes de la diversité biologique et les concepts biologiques importants de chacune de ces périodes historiques. Au cours de 3 grandes périodes, 
nous allons présenter comment l'évolution des représentations a façonné et influencé les opinions et l'imaginaire $d u$ grand public vis-à-vis du continent africain. La perspective historique de cette mise en scène de la flore et de la faune africaine permet, de notre point de vue, de dégager les clés de lecture nécessaires pour analyser les représentations ayant cours aujourd'hui. Puis, nous expliquerons que les représentations qui sont données à voir aux publics qui visitent ce nouveau musée sont le résultat d'un non-choix entre différents types de musées.

\section{La présentation de la diversité biologique à la période coloniale (1910-1958)}

5 C'est le Roi Léopold II qui fit construire le MRAC, un imposant édifice datant de 1910, inspiré par le Petit Palais à Paris. Les photos des salles d'exposition juste après l'inauguration montrent des vitrines avec de grands mammifères naturalisés présentés sur des socles dans des positions statiques. Les collections zoologiques étaient alors exposées selon les classifications en vigueur à l'époque: mammifères, anthropomorphes, oiseaux et mollusques, arthropodes, poissons, batraciens et reptiles (de Haulleville, 1910, p. 68-72).

6 Selon le directeur de l'époque, l'objectif était une «salutaire œuvre éducatrice des foules » et visait aussi bien les spécialistes que le grand public: "en observant les règles de la classification, on captive l'attention des hommes avertis; par l'art avec lequel on les présente, on arrête l'œil et l'attention du visiteur profane » (de Haulleville, A. 1910, p.6).

7 L'aspect menaçant des animaux vis-à-vis des humains (Figure 1) était également présent, par exemple pour les crocodiles du Nil : «On sait que ces gigantesques sauriens constituent le fléau des rivières africaines et sont la terreur des noirs parmi lesquels ils font de fréquentes victimes " (de Haulleville, 1910, p. 70). Si les humains restaient, à cette époque, absents des dispositifs muséographiques on voit progressivement apparaitre des évocations d'habitats autour des spécimens : sol naturel, branches... comme pour le groupe de gorilles de sexes et d'âges divers, très appréciés du public. En 1911, Émile Coart, alors directeur de la section de Sciences naturelles, reconnaissait que sans ce genre de groupe, un musée restait un «froid étalage d'objets inertes devant lequel le public défile indifféremment ». Mais il craignait que si les spécimens n'étaient pas bien documentés, on risquerait de présenter aux visiteurs des «fantaisies banales ", ou même un « spectacle forain » (Coart, 1911, p. 53). 


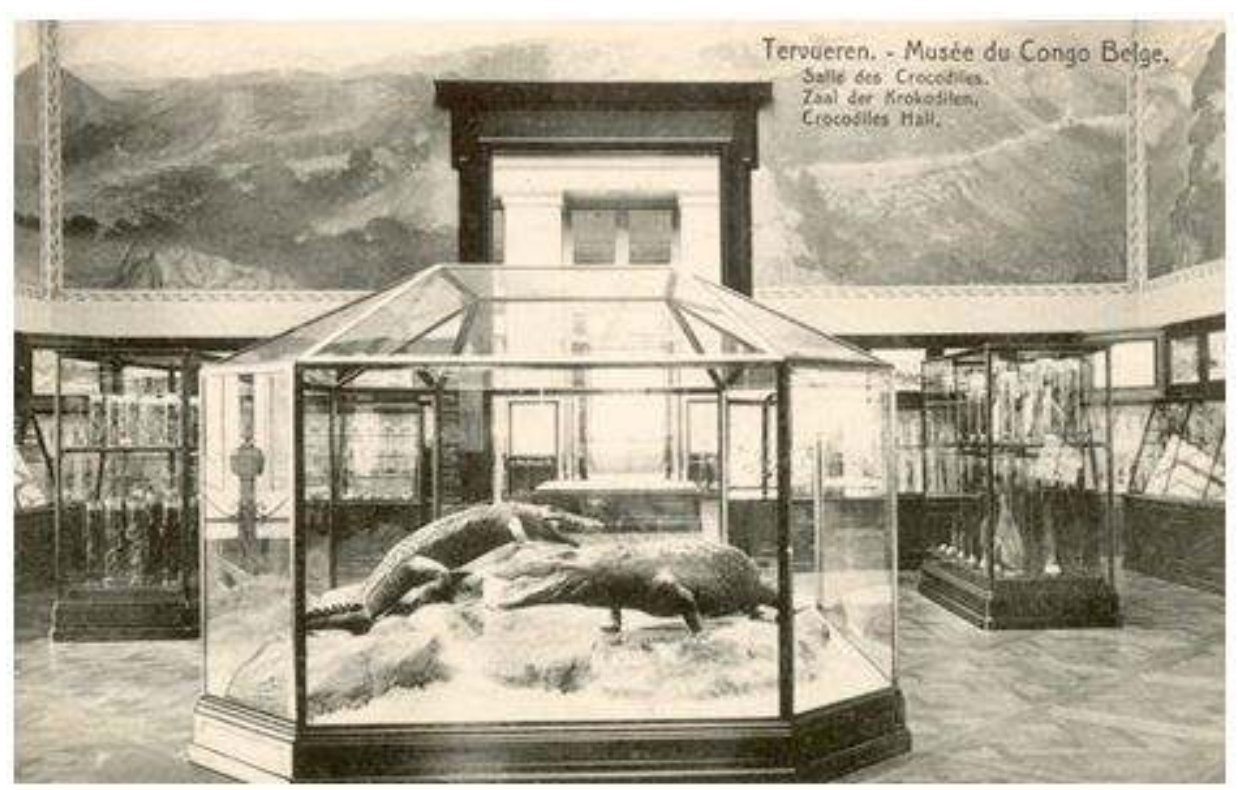

Figure 1. Carte postale (1910), représentant la « salle des crocodiles » du Musée du Congo Belge inauguré en 1910. (c) MRAC, Tervuren.

8 À la fin du $19^{e}$, les présentations zoologiques privilégiaient donc le côté sensationnel des prédateurs égorgeant leur proie. Compte tenu de l'engouement des Européens pour les safaris, de nombreux trophées de chasse furent légués et présentés au musée.

\section{L'exposition universelle de 1958 : La période moderne}

9 Le MRAC était représenté à l'exposition universelle de 1958, notamment par un «pavillon de la faune » situé au Heysel à Bruxelles, un site éloigné de Tervuren : les animaux naturalisés avaient été chassés en Afrique à cette occasion, et étaient présentés au sein d'un décor constitué d'une immense photo de paysage et d'éléments végétaux. Les spécimens ont ensuite été ajoutés dans les vitrines du musée (Sosson, 2008).

10 Suite à la prise en compte progressive des préoccupations écologiques, une quinzaine de dioramas zoologiques furent réalisés, dans les années 1970, pour contextualiser l'animal dans son habitat d'origine. Ces premiers dioramas avaient pour finalité de permettre une comparaison entre les quatre milieux écologiques du Congo: savanes septentrionale et méridionale, les forêts centrale et orientale (Couttenier, 2010, p. 137).

11 Alors même que ces différentes présentations muséographiques, apparues au cours du siècle passé, étaient toujours visibles au moment de la fermeture du musée fin 2013, des présentations plus récentes témoignaient de la prise en compte progressive d'aspects éducatifs contemporains. Ainsi, dès les années 1990, les membres du service éducatif ont mis en place des ateliers et des dispositifs pour aborder les aspects anthropiques dans les salles de biologie, introduisant progressivement les notions de protection de la nature avec notamment la Convention on International Trade in Endangered Species of Wild Fauna and Flora (CITES). En 2005, une exposition temporaire « Nature et culture » avait initié un dialogue entre différents domaines de recherche. Dans le catalogue, les responsables du musée revendiquaient même de jouer un rôle de médiateur entre 
politiciens et financeurs potentiels acteurs de la protection de la nature (Gryseels, 2004).

Enfin, la dernière version du guide de visite diffusée avant la fermeture présageait déjà certaines tendances de la rénovation: "Aujourd'hui, une prise de conscience écologique dicte de plus en plus notre manière de voir ces choses, et l'animal empaillé apparaît davantage comme un témoignage du passé » (Cappellemans, M. 2003, p.57).

13 Lors de la fermeture du MRAC au public, en 2013, ce sont plus de 15 millions de visiteurs qui avaient pu voir cette présentation d'une nature menaçante qu'il fallait donc dominer et contrôler. L'influence de cette exposition a été considérable puisqu'elle s'est prolongée « hors les murs ». En effet, le MRAC a souvent été représenté au cinéma et dans la littérature. L'exemple le plus connu étant peut-être Tintin au Congo, une bande dessinée que Hergé publia en 1930, remplie de clichés, et qui est toujours diffusée mondialement, avec un personnage inspiré de la statue de l'homme léopard exposée au MRAC (Figure 2), et dans laquelle Tintin fait exploser un rhinocéros et parade avec des défenses d'éléphant (Cappellemans, 2003, p.18).

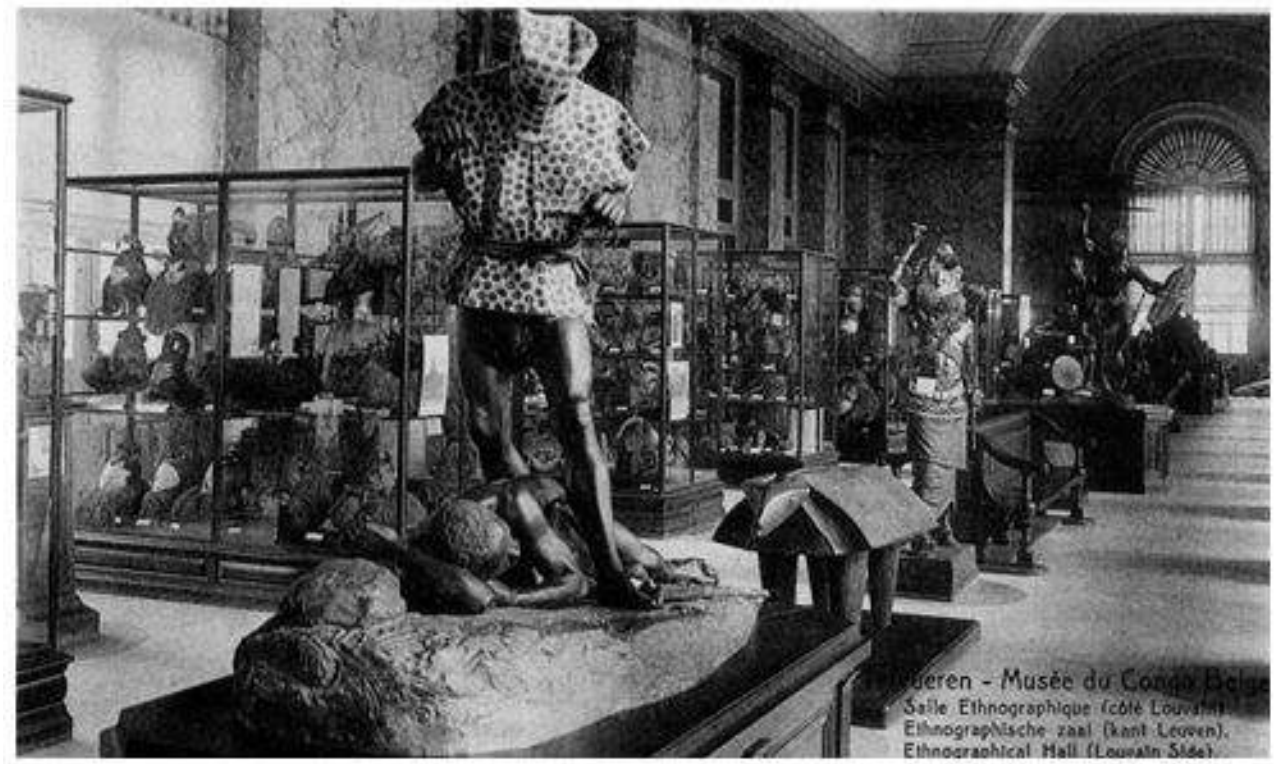

Figure 2. Carte postale (1910), représentant la « salle ethnographique » du Musée du Congo Belge inauguré en 1910. (c) MRAC, Tervuren.

14 Cette prestigieuse institution, qui incarnait le volet colonial de l'Histoire belge, est donc progressivement devenue un objet de controverses et de débats entre opinion publique, anciens coloniaux, membres de la diaspora, journalistes et experts internationaux.

\section{À partir de 2018 : après la rénovation}

Dans le musée rénové, conformément aux préconisations de l'équipe scientifique formulées dans les «Exhibition briefs » en 2011, le parcours est structuré comme un " voyage " à travers les biomes ${ }^{2}$ d'Afrique Centrale : en déambulant dans les salles, le visiteur découvre la diversité de paysages présents : savane et forêt dense, montagnes et volcans, lacs et fleuves, littoral (mangroves), tout cela entre deux zones désertiques au sud et au nord (Vande Weghe, J.P. 2004). Cet ordre de parcours correspond donc à la disposition réelle des biomes en Afrique Centrale vis-à-vis de l'Équateur. 
Dans un second temps, l'équipe scientifique a voulu faire connaître et faire comprendre aux visiteurs la dynamique de ces biomes, en insistant sur le fait qu'un biome n'est pas figé dans le temps, mais évolue sous l'effet de changements climatiques ou d'interventions humaines. Pour cela, l'équipe de muséologie a décidé de présenter des "focus", soit des exemples géographiques précis et réels au sein desquels ont eu lieu des missions de recherche menées par le MRAC et qui permettent de mettre en perspective, sur des études de cas, une évolution de ces rapports entre humains, nature et société (van der Hoorn, 2012). Il s'agissait de mettre fin à une vision trop stéréotypée des paysages africains comme c'était le cas auparavant avec la présentation de dioramas (Figure 3). À titre d'exemple, pour faire référence aux lacs africains, deux d'entre eux sont présentés. Le Lac Victoria a été retenu pour aborder les relations entre les espèces endémiques et une espèce exotique introduite dans les années 1950, la perche du Nil, ce qui a des conséquences sur la pêche et sur le développement socioéconomique des populations locales. Le Lac Tanganyika permet de présenter la très grande variété des cichlidés (présence de centaines d'espèces), ainsi que les divers modes de pêche.

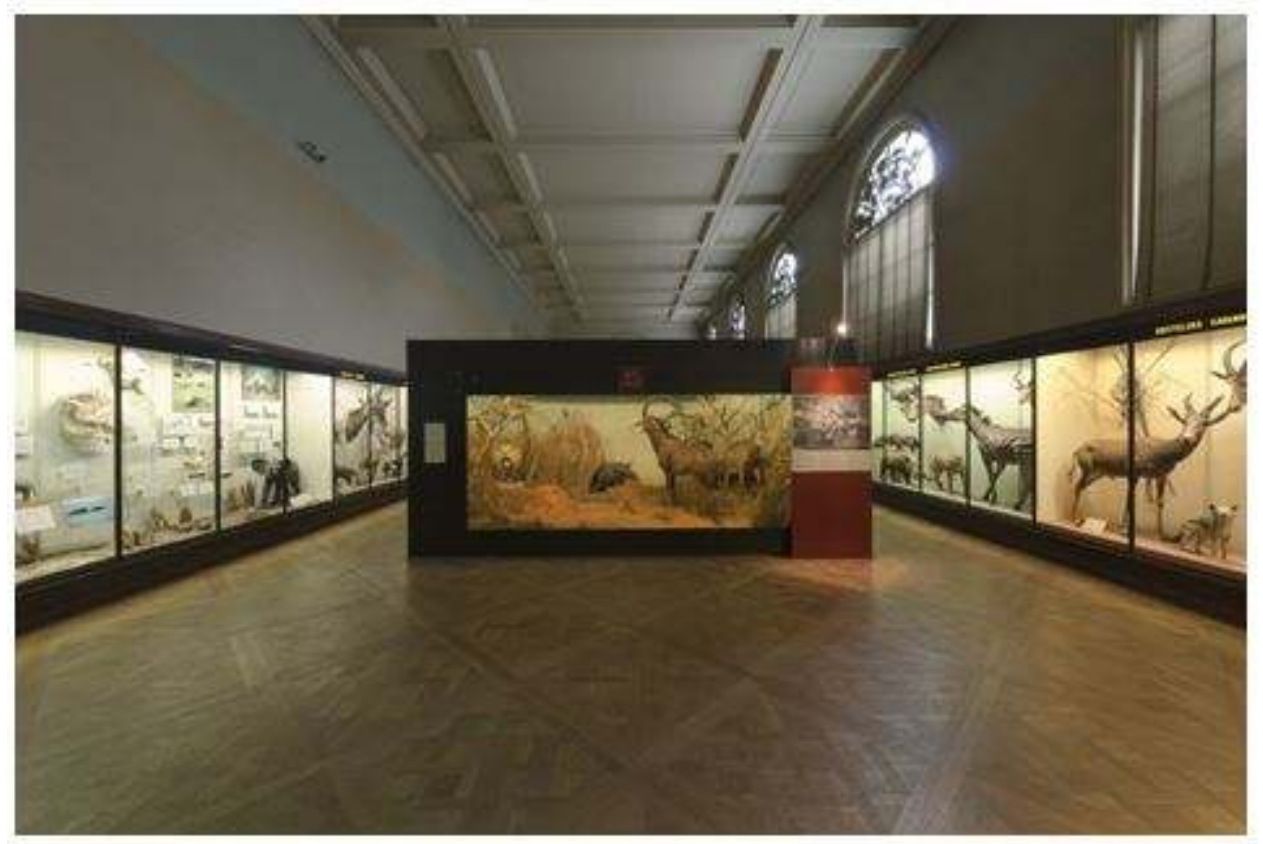

Figure.3. La salle de la section zoologie, avec les dioramas, juste avant la fermeture du musée pour rénovation, fin 2013 (c) Vandevijver, J. MRAC, Tervuren.

17 Au-delà de la découverte de la diversité des paysages culturels d'Afrique Centrale, l'équipe scientifique voulait souligner le fait que «La nature a un impact sur l'homme et l'homme agit sur la nature et s'en inspire", ce qui implique un passage d'une approche naturaliste vers une approche plus culturaliste de l'environnement. De plus, il s'agissait d'introduire le fait que la biodiversité était un concept hybride entre science et gouvernance (Girault et Alpe, 2011). La version définitive du scénario de l'exposition, un document établi en 2011 en interne, souligne ainsi que l'Afrique centrale a toujours été convoitée : "l'Afrique centrale est une région incroyablement riche et diverse, par la nature et la culture, mais c'est aussi, et depuis longtemps, un objet de convoitise. Les collections et l'histoire du musée témoignent de cette richesse et de cette convoitise». 
Si depuis son ouverture, le MRAC a toujours eu pour vocation de présenter le territoire du Congo et ses richesses, la muséographie retenue au cours des 3 périodes, ci-dessus définies, ainsi que l'ensemble des contenus et des objets exposés ont évolué selon la représentation dominante à chacune de ces époques sur la faune, la flore et les écosystèmes du Congo. Durant chacune de ces périodes, cinq thèmes ont été constamment abordés pour décrire ce qu'est une écorégion et illustrer le développement du territoire : le territoire (dont l'espace géographique et les paysages), la diversité biologique (la faune et la flore), les populations locales (les humains), les ressources, la puissance coloniale (la Belgique). En revanche, l'importance relative de chacun de ces thèmes durant les périodes a évolué au gré des attentes de la société et de son éthos. Ainsi, on peut noter une évolution d'une représentation idéalisée, sensée enthousiasmer les visiteurs, vers une représentation plus rationnelle, destinée à faire prendre conscience aux visiteurs de la situation menacée de la nature (Tableau 1).

Tableau 1. Présentation des concepts et expots utilisés pour illustrer les différents thèmes expliquant la biodiversité, en fonction des différentes périodes muséographiques

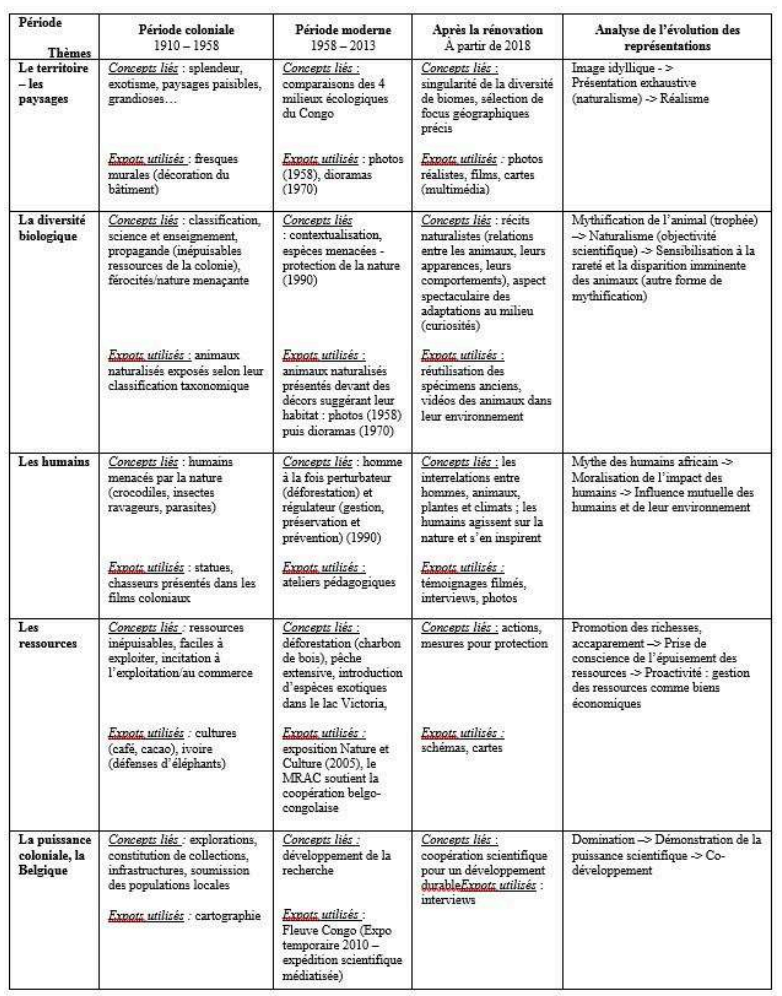

\section{L'Africamuseum : une juxtaposition de quatre conceptions du musée}

Durant toute la rénovation, soit pendant 8 ans environ, l'équipe s'est heurtée à des obstacles majeurs de différentes natures, à la fois matériels (le bâtiment " connoté », les collections datées) que disciplinaires (présupposés épistémologiques et approches monodisciplinaires). Ces obstacles, que nous allons décrire ci-dessous, ont dû être dépassés, voir contournés pour offrir une vision contemporaine de l'Afrique centrale et de ses paysages culturels, au sein desquels les humains occupent un rôle essentiel. 
20 Pour cela, l'équipe de rénovation s'est appuyée sur différents types ou identités de musées, satisfaisant alternativement des aspects de certains des quatre concepts de musées que nous avons retenus pour notre analyse. Nous verrons qu'un compromis entre ces quatre concepts de musées a pu être réalisé

21 À l'Africamuseum, c'est le bâtiment entier qui « respire " l'histoire coloniale. Il s'agit d'un monument classé et le projet de rénovation était basé sur le principe du respect de «l'esprit des lieux» (Viel, 2001). Ainsi, les peintures murales, les inscriptions, les sols en marbre ont dû rester dans l'état initial. Le mobilier d'origine, les dizaines de vitrines murales ou centrales, alignées, sur socles en bois, ont été restaurés à l'identique. La grande majorité des collections zoologiques, et donc leur mode de naturalisation, témoignent de la période des collectes débridées des coloniaux en Afrique. Comment prétendre alors vouloir modifier le rapport à l'environnement des visiteurs, alors même qu'avant d'entrer dans l'exposition permanente, ils sont largement influencés par l'influence architecturale de ce bâtiment?

\section{Le musée d'un musée colonial de sciences}

Un premier parti pris muséologique a donc été imposé par la réutilisation du bâtiment et des collections: le musée d'un musée colonial de sciences. Celui-ci s'exprime très clairement dans la Salle des crocodiles, une salle qui a été restaurée, à partir de cartes postales et d'anciens textes d'exposition, à l'identique de son état initial en 1910. Selon une journaliste du Point, il s'agit d'une façon pour le musée de porter un regard réflexif et un recul critique sur lui-même: "Dans le même louable esprit d'autocritique, la fameuse salle des Crocodiles exposée comme à l'ancienne devient l'occasion de réfléchir au regard porté en 1920 sur la nature congolaise et l'« Afrique exotique et romantique» (Marin La Meslée, 2018). Ce regard critique est matérialisé par une barrière transparente : "De manière symbolique, un écran sépare cette salle des autres pour indiquer (...) qu'elle ne reflète pas notre vision actuelle de l'Afrique », explique Guido Gryseels (Belga News, 2018).

Lors du processus de rénovation, l'option de conserver le musée dans son état d'origine comme un musée d'un musée, a souvent été évoquée. Selon Gryseels, cette idée de "garder l'exposition de l'ancien musée telle qu'elle était, comme une sorte de témoignage du colonialisme et du regard colonial, et de construire en sus un nouveau musée sur l'Afrique contemporaine " était intéressante, mais a dû être abandonnée pour des raisons budgétaires (Lismond-Mertes, 2019). Dans le même esprit, on aurait pu décider de conserver les mises en scènes, c'est-à-dire les dioramas en entier. Mais les spécimens en ont été extraits et disposés dans un contexte dénué de nouveau discours. Ce choix a été orienté par la volonté scénographique de mettre le musée au goût du jour, d'enlever au maximum les séparations par des vitres entre les spécimens et le public. Mais aussi parce qu'en présentant des biomes géographiques précis, de nouveaux ensembles d'espèces devaient être composés. Dans la scénographie contemporaine, les spécimens sont exposés de façon esthétisante avec pour évocation de leur habitat de grandes photos de paysages. 


\section{Le musée encyclopédique}

La tension entre le souhait de représenter fidèlement l'institut scientifique de recherche et d'offrir aux visiteurs une image de l'Afrique contemporaine est restée un souci permanent. Une première volonté a été celle d'adopter le principe du muséeencyclopédique: dans les textes d'exposition, les chercheurs du MRAC ont documenté des thèmes d'ordre biologique : la nidification, le camouflage, la chaîne alimentaire, l'endémisme... Or en 2013, suite à une évaluation jugeant le scénario trop encyclopédique, l'exposition a davantage été conçue comme un collier de perles, c'està-dire avec des salles ayant chacune une identité propre afin que les visiteurs soient surpris, émus. Ainsi, la salle «forêt tropicale » se distingue par son approche immersive avec de très grandes photographies et une scénographie qui évoque les grands arbres (Figure 4). L'autre salle, qui présente le désert, la savane, les lacs et les montagnes, est structurée autour de grands podiums centraux composés également de grands décors de photos et d'ensembles d'animaux. L'approche éducative a pour objet de « faire voir et émouvoir » le visiteur par la présentation de belles photos de chacun des biomes, des paysages et animaux impressionnants, pour ensuite «faire connaître et comprendre » et enfin l'inciter à se forger sa propre opinion, à réfléchir (Quertier et Girault, 2011).

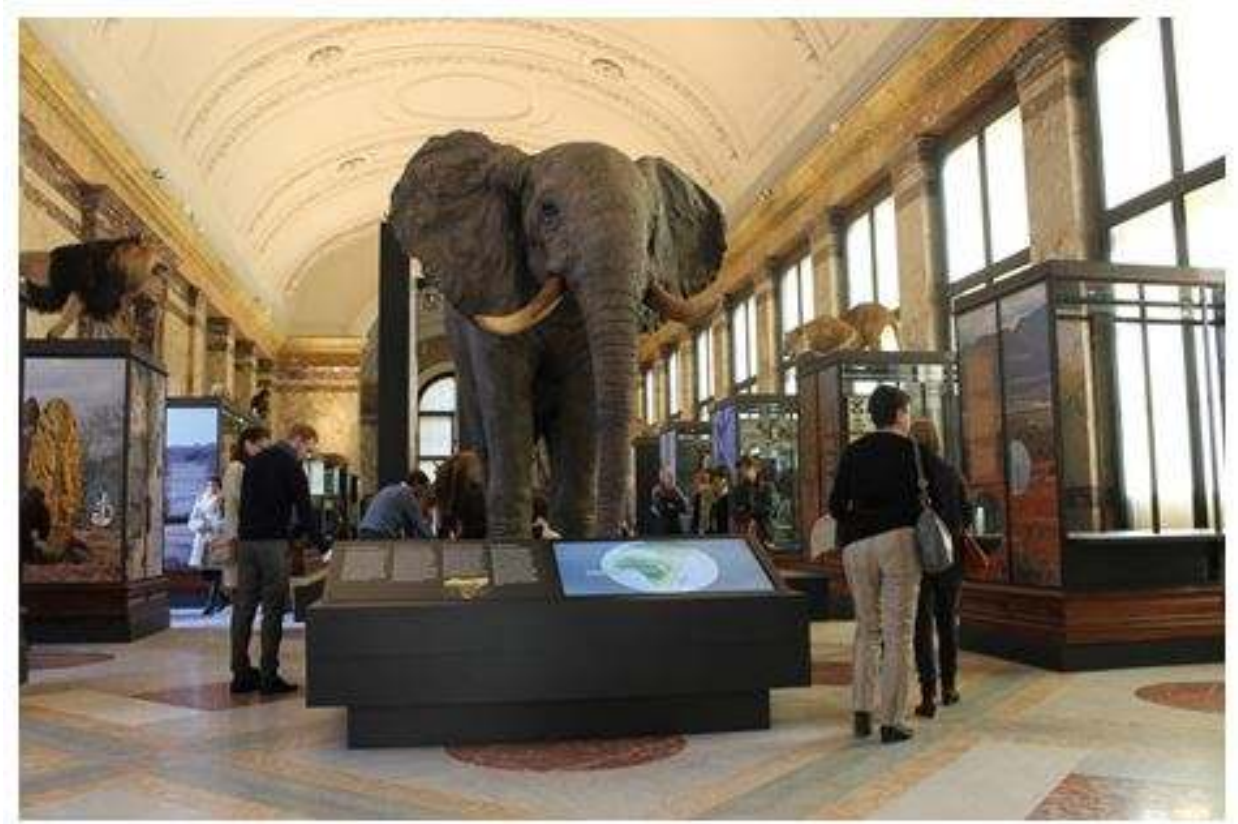

Figure.4. Le podium « savane » et les félins en haut des vitrines dans la salle Paysages et biodiversité Africamuseum, 2019 @ van der Hoorn, S

\section{Le musée forum}

C'est l'idée du musée-forum qui exprime cependant le principal objectif de la rénovation tel qu'il était formulé dès les premiers scénarios: offrir aux visiteurs une vision de l'Afrique centrale d'aujourd'hui, où les humains occupent une place centrale. En faisant intervenir dans l'exposition à la fois des chercheurs du MRAC et des interlocuteurs locaux, experts ou habitants de la nature africaine, différentes approches coexistent. On présente des questions qui font débat pour montrer la nécessaire recherche « d'un équilibre entre la protection des richesses en Afrique Centrale et les besoins vitaux et économiques d'une population croissante $»^{3}$. Cela se traduit principalement par des 
témoignages et des interviews sur des écrans répartis à travers l'exposition. Par exemple, dans la vitrine des Parcs nationaux, on montre sur différents écrans le point de vue d'acteurs : habitant, exploitant, gestionnaire sur l'avenir du Parc des Virunga. La vision est celle de l'humain qui apparaît à la fois comme l'élément perturbateur, mais aussi comme "l'élément régulateur qui va gérer, préserver et prévenir " (Alpe et Girault, 2011).

Or, ce type de musée était difficile à réaliser compte tenu de la nature de l'institution au sein de laquelle les sections de recherche en sciences humaines et en sciences naturelles fonctionnent le plus souvent séparément, ce qui n'interdit pas à certains chercheurs de s'associer pour des projets communs. Ainsi, les chercheurs en zoologie effectuent des recherches en taxonomie principalement, et les aspects socioéconomiques d'une région naturelle ou les interactions avec les humains ne constituent pas le centre de leur travail. Dans ce contexte historique de cloisonnement disciplinaire au MRAC, la notion hybride de gouvernance de la biodiversité n'est pas réellement étudiée et, en conséquence, elle est peu abordée dans la trame narrative de l'exposition.

\section{Le musée - lieu de divertissement}

Enfin, l'aspect musée - lieu de divertissement est également présent. S'adressant principalement à un public de non-spécialistes (visites familiales ou scolaires), le musée perçu comme lieu de divertissement ne pouvait être négligé. En effet, l'une des finalités des expositions permanentes et temporaires des musées est de présenter les collections aux publics et principalement les plus gros spécimens. Dans les grands musées qui ont fermé, de nombreux visiteurs s'attendent à retrouver certaines présentations spectaculaires qu'ils ont vues dans leur enfance et qu'ils veulent faire découvrir à leurs enfants ou petits-enfants. Dans un grand musée d'Histoire naturelle, le muséologue est donc contraint de présenter le maximum d'animaux naturalisés. Cependant, l'image d'abondance, de diversité d'espèces animales est, de nos jours, contraire au message lié à la crise de la biodiversité, de l'appauvrissement des biomes menacés par l'effet du climat et des activités humaines. Les scientifiques précisent en effet que, de nos jours, « les forêts du Congo sont vides » suite à la chasse et au braconnage. Comment pouvoir prétendre présenter l'absence d'espèces au sein d'un musée d'Histoire naturelle qui est basé, à l'opposé, sur la présentation et la valorisation de ses collections ? Dans le musée rénové, certes le visiteur va percevoir des spécimens élevés au rang d'objets de musée (Figure 5), soigneusement présentés dans un décor dénudé, mais il verra également une grande quantité d'animaux, grands et petits, représentant chacun des milieux naturels. 


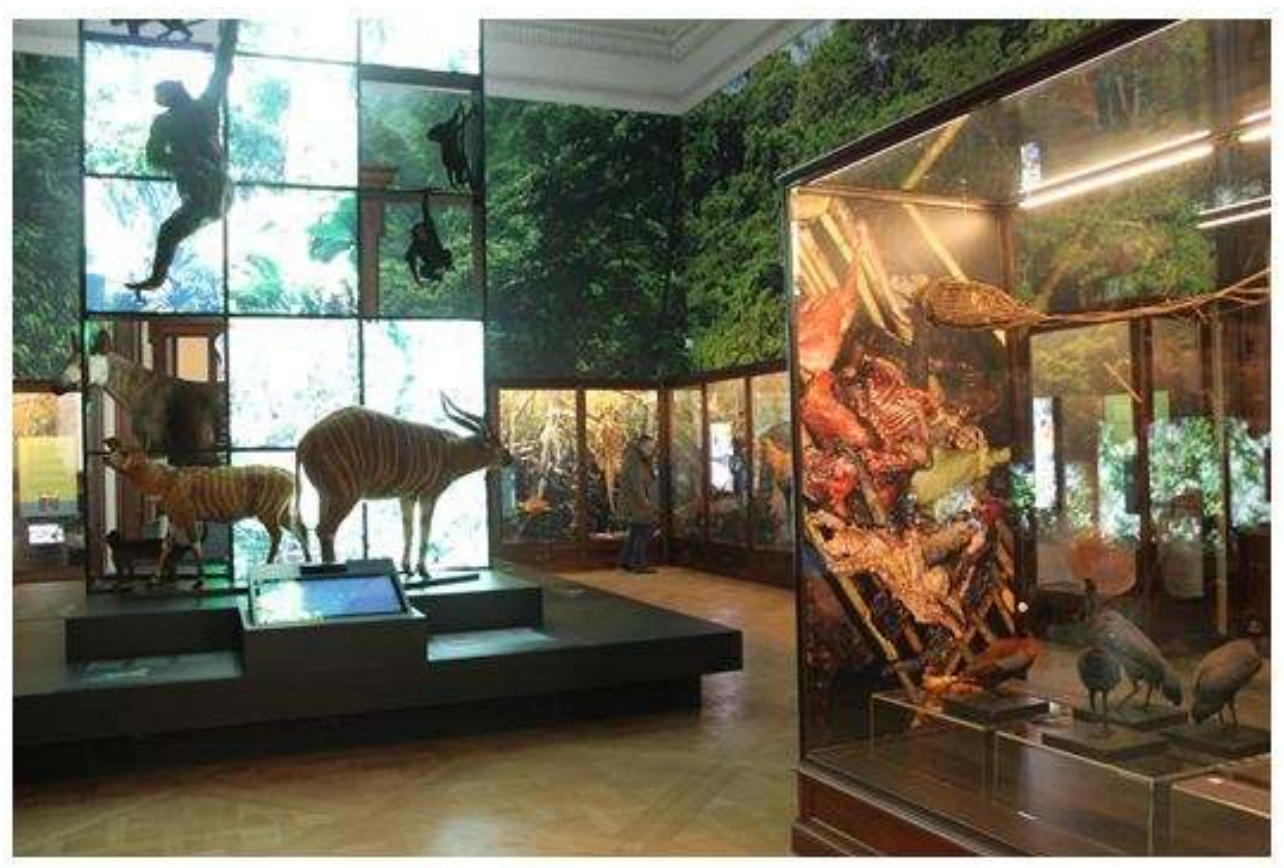

Figure .5. La salle « forêt tropicale » de la zone Paysages et biodiversité, Africamuseum, 2019 @ van der Hoorn, $S$.

Pour valoriser leurs attitudes dynamiques et dans une logique de scénographie de spectacle, des félins ont été disposés, sans le moindre souci d'intégration dans la trame narrative de l'exposition, en haut de vitrines. Ces spécimens anciens, dont certains font partie du vécu de nombreux visiteurs, traduisent un rapport à l'environnement, passé ou actuel, qui devrait également être expliqué aux visiteurs. En effet, depuis l'origine du musée, si les animaux mis en scène constituaient souvent un premier contact avec la nature africaine "exotique", ils sont progressivement devenus des pièces emblématiques du musée colonial belge pour plusieurs générations de visiteurs. Il en est ainsi des grands mammifères, mais aussi de la pirogue et la statue de l'hommeléopard reprise dans l'album de Tintin au Congo.

Différentes conceptions de musée coexistent donc dans la présentation actuelle des salles Paysages et biodiversité. Chaque conception résulte soit de l'héritage matériel du musée, soit des objectifs de l'équipe de conception et de ses conseillers. Dans le Tableau 2, nous montrons que chaque type de musée présente à la fois des avantages et des inconvénients dans la transmission du discours au public. 
Tableau 2 : Analyse comparée SWOT ${ }^{4}$ de différents types de musées, composant la présentation actuelle des espaces Paysages et biodiversité à l'Africamuseum

\begin{tabular}{|c|c|c|c|c|}
\hline & Points forts & Points faibles & Opportumités & Menaces \\
\hline Musée de musée & $\begin{array}{l}\text { Description } \\
\text { historique fidèle et } \\
\text { complète }\end{array}$ & $\begin{array}{l}\text { Point de vue } \\
\text { colonial unique }\end{array}$ & $\begin{array}{l}\text { Aggiornamento sur } \\
\text { la période } \\
\text { coloniale }\end{array}$ & $\begin{array}{l}\text { Glorification du } \\
\text { passé colonial / } \\
\text { désintérêt de } \\
\text { l'Afrique } \\
\text { d'aujourd'hui- } \\
\text { perte du lien avec } \\
\text { l'actualité }\end{array}$ \\
\hline Musée-forum & $\begin{array}{l}\text { Pont vers } \\
\text { l'Afrique }\end{array}$ & $\begin{array}{l}\text { Difficulté de } \\
\text { représenter le } \\
\text { présent (en } \\
\text { mouvement) }\end{array}$ & $\begin{array}{l}\text { Ouverture aux } \\
\text { Africains, } \\
\text { Musee vecteur de } \\
\text { messages } \\
\text { politicues }\end{array}$ & $\begin{array}{l}\text { Effacement du passé } \\
\text { colonial (amnésie), } \\
\text { présentéisme, } \\
\text { instrumentalisation }\end{array}$ \\
\hline $\begin{array}{l}\text { Musée- } \\
\text { divertissements }\end{array}$ & $\begin{array}{l}\text { Attractivité, } \\
\text { Scénographie } \\
\text { innovante }\end{array}$ & $\begin{array}{l}\text { Débauche } \\
\text { technologique } \\
\text { purement } \\
\text { sensorielle }\end{array}$ & Intérêts des publics & Superficialité \\
\hline $\begin{array}{l}\text { Musée- } \\
\text { encyclopédie }\end{array}$ & $\begin{array}{l}\text { Etat des lieux de } \\
\text { la connaissance } \\
\text { actuelle }\end{array}$ & $\begin{array}{l}\text { Présentation } \\
\text { indigeste, trop } \\
\text { d'information }\end{array}$ & $\begin{array}{l}\text { Valorisation de } \\
\text { l'institut de } \\
\text { recherche }\end{array}$ & $\begin{array}{l}\text { Exclusion de } \\
\text { certains publics ou } \\
\text { de catégories socio- } \\
\text { professionnelles }\end{array}$ \\
\hline
\end{tabular}

\section{Conclusion : Un compromis « à la belge »}

Finalement, les juxtapositions muséales issues de la rénovation du Musée Royal de l'Afrique Centrale sont appréciées comme un «bon compromis »; soit d'après Geert Vanistendael, «un compromis où personne n'est content» (Mandy, 2014). De nombreux articles de presse ont en effet mis en évidence les frustrations des différentes parties prenantes après l'inauguration en décembre 2018 de l'Africamuseum. Les compromis muséologiques qui ont été trouvés pour chaque vitrine et,ou chaque espace à scénographier et qui s'intègrent tantôt dans l'un ou l'autre des concepts de musées sont une des sources de ce mécontentement.

Il en résulte finalement un désordre global éloignant une hypothétique unité muséale.

Enfin, la principale critique après l'inauguration du musée est de ne pas avoir décolonisé le musée. Par exemple dans la salle « Paysages et biodiversité », le discours est toujours très eurocentré dans la mesure où c'est majoritairement un regard belge, européen, qui est posé sur la nature africaine. Dans un sens, le MRAC est resté au plus près de son identité : un centre d'étude de l'Afrique centrale. Les objets qu'il présente sont issus de sa propre histoire et le discours a été écrit par les membres de son personnel.

1 Or, tout au long du processus, on a pu ressentir une volonté, une ouverture à ouvrir le dialogue, à aligner le MRAC dans une tendance de remise en question de relations mondiales en pleine évolution.

Différents partis pris ont été avancés par des collaborateurs au fil du temps, qui auraient nécessité un changement radical de la muséologie. Ainsi, Wastiau, ancien collaborateur et commissaire d'exposition en 2000, soutenait une approche où les conséquences de la colonisation sur l'Afrique centrale contemporaine ne seraient pas seulement abordées dans les salles de la section historique, mais aussi dans Paysages et biodiversité : « Les problèmes climatiques, écologiques et humains d'aujourd'hui sont la 
conséquence directe $\mathrm{du}$ colonialisme et du post-colonialisme. C'est d'une telle évidence » (Lismond-Mertes, 2019, p.41).

En 2000, Capenberghs, chargé du projet de modernisation muséologique, évoquait la nécessité d'accompagner chaque objet de trois histoires différentes : " un point de vue artistique, un point de vue sur la "vie sociale" de l'objet, ou encore en exprimant ce qu'évoque l'objet pour un Congolais d'aujourd'hui ». Selon lui, ces « plateformes multiinterprétables " correspondraient mieux à un musée multi-disciplinaire (LismondMertes, 2019, p.48). Il insiste aussi sur la nécessité de créer des «miroirs et des fenêtres" dans ce genre de musée, allant même jusqu'à intégrer au futur Africamuseum une section Musée de la Belgique qui aurait été conçue par des Africains, dans l'objectif de "rendre visible l'utilisation de clichés et de fausses catégories " (Lismond-Mertes, 2019, p. 48).

Ainsi, plutôt qu'un changement radical, on peut considérer la rénovation du MRAC comme un premier face-à-face avec soi-même. Comme le disait Gryseels à l'occasion de l'une des conférences de presse précédant l'inauguration, la décolonisation est une affaire de deux générations : "C'est un processus global, dont on ne pourra mesurer les effets que dans une dizaine d'années. Nous avons fait un pas en ce sens, mais il y a encore beaucoup de travail à faire » (Lismond-Mertes, 2019, p. 29).

En attendant, dans les salles de Paysages et biodiversité, il est probable que seuls les visiteurs qui lisent très attentivement les textes percevront l'ampleur de cette grande remise en question. D'autres visiteurs éprouveront simplement du plaisir à admirer les spécimens de leur enfance.

\section{BIBLIOGRAPHIE}

Belga News. AfricaMuseum, un nouveau musée de l'Afrique, plus critique de la colonisation. RTBF.be Culture, 10 décembre 2018 à 11 h 15.

De Haulleville, A. (1910). Le Musée du Congo Belge à Tervueren. Bruxelles : Imprimerie Ve Monnom,

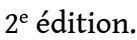

Cappellemans, M. (2003). La Clé du Musée. Guide du visiteur du MRAC. Tervuren : Musée royal de l'Afrique centrale.

Coart, E. (1911). Mission Coart en Allemagne, Rapport sur un voyage d'études en Allemagne (du 13 au 28 septembre 1911)

Couttenier, M. (2010). Si les murs pouvaient parler. Le Musée de Tervuren. Tervuren : Musée royal de l'Afrique centrale.

Girault, Y. et Alpe, Y. (2001). La biodiversité, un concept hybride entre science et gouvernance. Dans Legardez, A. Simonneaux, L. (Coord.). Développement durable et autres questions d'actualité. Questions socialement vives dans l'enseignement et la formation (p. 1-20). Dijon : Éditions Educagri Gryseels, G. (2004). Préambule. Dans Bashige E. Nature et culture en République démocratique du Congo. Bruxelles : Musée royal de l'Afrique centrale. 
Lismond-Mertes, A. (2019). « Nous connaissons un succès spectaculaire. » Dossier Tervuren, décolonial ?. Ensemble ! Pour la solidarité, contre l'exclusion. Quadrimestriel, 99, 24-31.

Lismond-Mertes, A. (2019). « Tervuren reste un lieu de fausses mémoires. » Dossier Tervuren, décolonial ?. Ensemble ! Pour la solidarité, contre l'exclusion. Quadrimestriel, 99, 39-46.

Lismond-Mertes, A. (2019). « Il faut une muséologie multivocale. » Dossier Tervuren, décolonial ?. Ensemble! Pour la solidarité, contre l'exclusion. Quadrimestriel, 99, 47-48.

Mandy, M. (2014). Oui, mais non. Le compromis à la belge. Film documentaire. Lunablue Film à Bruxelles.

Marin La Meslée, V. (2018). Africa Museum de Bruxelles, un vaste chantier ! Le Point.fr, 9 décembre 2018.

Quertier, E. et Girault, Y. (2011). Tendances actuelles de la mise en exposition de la biodiversité. Dans Alpe, Y. et Girault, Y. (dir.), Éducation au développement durable et à la biodiversité : concepts, questions vives, outils et pratiques. Actes du colloque (p.34-57). Digne-les-Bains : IUT de Provence. Sosson, P. (2008). Le pavillon de la faune, expertises croisées et créations d'ambiances. Travail de fin d'études inédit. ISACF La Cambre à Bruxelles.

Van der Hoorn, S. (2012). La rénovation du Musée royal de l'Afrique centrale. Dans Girault, Y. et Van-Praët, M. (dir.), MuséoMuséum, 20 ans d'enseignement de la muséologie au Muséum national d'Histoire naturelle. Actes du colloque (p.269). Paris : Muséum national d'Histoire naturelle.

Vande Weghe, J.P. (2004). Forests of Central Africa. Nature and man. Pretoria : Protea, $2^{\mathrm{e}}$ édition.

Viel, A. (2001). Quand souffle l'esprit des lieux. Actes du colloque « Médiation culturelle dans un lieu patrimonial en relation avec son territoire », 6 et 7 décembre 2000. Saint-Vougay : Association pour l'animation du Château de Kerjean.

\section{NOTES}

1. https://www.africamuseum.be/

2. Vaste région biogéographique s'étendant sous un même climat (Larousse)

3. Document interne d'avant-projet, datant du 12 novembre 2014, traduit du néerlandais par Sabine van der Hoorn

4. Analyse SWOT: strengths (forces), weaknesses (faiblesses), opportunities (opportunités), threats (menaces)

\section{RÉSUMÉS}

La rénovation du Musée Royal de l'Afrique Centrale de Belgique, aujourd'hui appelé Africamuseum, a été l'occasion d'analyser comment culture et nature africaines ont été présentées pendant plus d'un siècle. Musée colonial conçu au début du XXe siècle et rouvert en 2018, l'Africamuseum a été le lieu de divers modes d'utilisation des pièces d'exposition, influençant ainsi les opinions et l'imaginaire du grand public belge vis-à-vis du continent 
africain. En adoptant la perspective historique sur la mise en scène de la flore et de la faune africaine, un éclairage particulier peut être apporté sur la muséologie développée à l'occasion de la rénovation. Il apparaît en effet que les représentations ayant cours dans le musée rénové, empruntent des caractéristiques de différents types de musées («musée de musée », "muséeforum », «musée-divertissements», «musée-encyclopédie») illustrant un non-choix muséologique, que certains appelleraient « un compromis à la belge ».

The renovation of the Royal Museum for Central Africa in Belgium, now named Africamuseum, was an opportunity to analyze how African culture and nature have been presented for more than a century. Conceived as a colonial museum in the beginning of the $\mathrm{XX}^{\text {th }}$ century, the Africamuseum evolved as the exhibits, thus influencing the opinions and the imagination of the Belgian public towards the African continent. By adopting an historical perspective on the exhibition of African flora and fauna, a particular light can be shed on the museology developed during the renovation in 2018. Indeed, it appears that the representations taking place in the renovated museum aggregates characteristics from different types of museums ("museum-of-amuseum", "museum-forum", "museum-entertainment", "museum-encyclopedia") illustrating a non-museological choice, which some would call "a Belgian compromise".

\section{INDEX}

Mots-clés : biodiversité, muséologie, histoire, musées, Africamusuem Index géographique : Belgique

Keywords : biodiversity, museology, history, museum, Africamuseum

\section{AUTEUR}

\section{SABINE VAN DER HOORN}

Sabine van der Hoorn est une muséologue et scénographe indépendante spécialisée dans la conception d'expositions scientifiques et de projets culturels. De 2011 à 2019, elle a exercé la fonction de Chargée de projet de la rénovation de la zone « Paysages et biodiversité » à l'Africamuseum (Tervuren, Belgique). Formée à la scénographie à l'École des Arts d'Utrecht (Pays-Bas), elle est titulaire d'un Master en Muséologie du Muséum National d'Histoire Naturelle de Paris (France). Ses différentes réalisations sont accessibles sur www.scenoscience.com 